\title{
HEMATOMA SUPRARRENAL POR ROTURA DE MIELOLIPOMA. A PROPÓSITO DE UN CASO
}

\author{
G. SUÁREZ ARTACHO*, J. RODRÍGUEZ MUÑOZ*, M.Á. GÓMEZ BRAVO*, \\ P. CAMPOY MARTÍNEZ**, F. LÓPEZ BERNAL* \\ *Servicio de Cirugía General y Aparato Digestivo. **Servicio de Urología. \\ Hospital Universitario Virgen del Rocío. Sevilla.
}

Actas Urol Esp. 28 (10): 785-788, 2004

\section{RESUMEN}

HEMATOMA SUPRARRENAL POR ROTURA DE MIELOLIPOMA. A PROPÓSITO DE UN CASO

El mielolipoma es un tumor benigno de la corteza suprarrenal, no funcionante y normalmente asintomático, que suele diagnosticarse de forma incidental en pruebas de imagen. La rotura y sangrado del mielolipoma es una complicación poco habitual, que puede concluir en la organización de un hematoma o, raramente, ocasionar hemorragia retroperitoneal masiva. La compresión de estructuras adyacentes por hematomas retroperitoneales (HR) de origen adrenal, es un evento poco frecuente. La indicación quirúrgica del HR continúa siendo, hoy día, una decisión difícil y controvertida, pues suele resolverse con tratamiento conservador y actitud expectante.

Presentamos el caso de un varón de 69 años de edad, que acudió a Urgencias por dolor abdominal de 7 días de evolución, cuyo inicio coincidió con traumatismo leve. Las pruebas de imagen revelaron la existencia de hematoma retroperitoneal derecho de $16 \mathrm{~cm}$ de posible origen adrenal, con signos de sangrado activo, que colapsaba y desplazaba vena cava. Tan sólo hemos encontrado 1 caso descrito en la literatura de compresión de vena cava secundario a hematoma dependiente de glándula suprarrenal.

En nuestro caso fue necesaria laparotomía urgente, suprarrenalectomía derecha y exéresis del hematoma.

PALABRAS CLAVE: Mielolipoma. Hematoma retroperitoneal. Glándula suprarrenal.

\section{ABSTRACT}

ADRENAL GLAND HEMATOMA DUE TO RUPTURE OF MYELOLIPOMA. A CASE REPORT

The myelolipoma is a benign tumour of the adrenal cortex, non functioning and normally asymptomatic, which can be diagnosed by chance in imaging test. Rupture and bleeding of the myelolipoma is an infrequent complication, which can conclude with the formation of a haematoma or, less usually, result in a massive retroperitoneal haemorrhage. The compression of adjacent structures by retroperitoneal haematomas with adrenal origin is a possible but not frequent eventuality.

Indications for surgery of haematoma retroperitoneal continue to be, nowadays, a difficult and controversial decision, because the problem is normally resolved with conservative treatment and a watchful approach.

We are presented with a 69 year old male who came to casualty with abdominal pain of 7 days duration, whose origin coincided with a light blow. The image test revealed the existence of a retroperitoneal haematoma with adrenal gland origin of $16 \mathrm{~cm}$, with active bleeding, which was displaced and compressed the cava vein. We have only found one case, in the literature, of compression of the cava vein coused by adrenal gland haematoma. In the case under our observation, urgent surgery was needed. 
$\mathrm{E}$ 1 mielolipoma de glándula suprarrenal es un tumor benigno de la corteza poco frecuente, no funcionante endocrinológicamente, que se origina a partir de tejido adiposo maduro con focos de células hematopoyéticas.

Suele observarse en individuos de edad media o avanzada, como hallazgo incidental de pruebas de imagen o autopsias (0,08-0,4\%). Este tipo de diagnósticos, ha sido favorecido en los últimos años por el aumento del número de exploraciones radiológicas realizadas y el mayor poder resolutivo de éstas ${ }^{1}$.

Son, normalmente, lesiones menores de $4 \mathrm{~cm}$ clínicamente silentes. Cuando superan dicho tamaño pueden ocasionar disconfort o dolor abdominal $^{2}$.

El sangrado por rotura de mielolipoma, es una complicación poco frecuente que, de producirse, suele ser autolimitado y de escasa cuantía, organizándose en HR. En raras ocasiones, llega a ser causa de hemorragia retroperitoneal masiva ${ }^{3}$. Tales hematomas, generalmente pasan desapercibidos por lo que, al igual que el mielolipoma, se descubren de forma casual en pruebas de imagen tras largo tiempo de evolución ${ }^{1,4}$. En tales casos y sobre todo, cuando son mayores de $4 \mathrm{~cm}$, pueden ser radiológicamente indistinguibles de neoplasias, obligando a realizar intervenciones invasivas como PAAF, biopsias guiadas por TAC o incluso lumbotomías exploradoras.

\section{CASO CLÍNICO}

Paciente de 69 años de edad que, como antecedentes personales, presentaba adenocarcinoma de próstata bien diferenciado tratado con análogos de LHRH y mielolipoma de glándula suprarrenal derecha de $2,5 \mathrm{~cm}$ diagnosticado casualmente mediante TAC realizado 6 años atrás (Fig. 1).

Acudió a nuestro servicio de Urgencias por astenia, anorexia, fiebre de $38,0^{\circ} \mathrm{C}$, y dolor abdominal de una semana de evolución coincidiendo, el inicio del cuadro, con caída accidental de cama. A la exploración, el paciente presentaba regular estado general aunque hemodinámica estable. El abdomen era globuloso y se encontraba ligeramente distendido. A la palpación se objetivó dolor en el hipocondrio derecho con signo de Murphy positivo. La auscultación cardio respiratoria resultó normal.

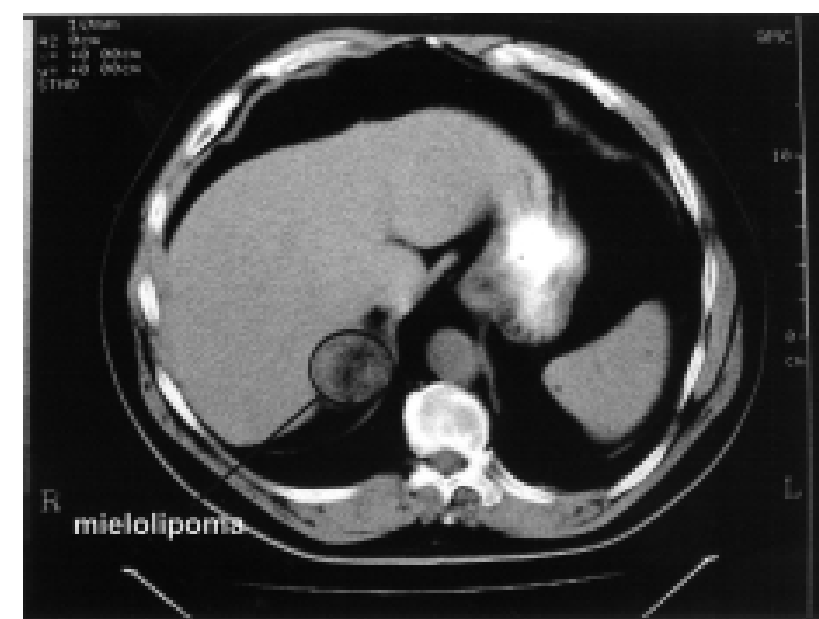

FIGURA 1. Diagnóstico incidental de mielolipoma en TAC de control.

El hemograma mostraba leucocitosis de $13.800 * 10$ e9/L y neutrofilia (83\%), hemoglobina de $110 \mathrm{~g} / 1 \mathrm{y}$ bilirrubina de $1,5 \mathrm{mg} / \mathrm{dl}$ a expensas de la indirecta $(0,9 \mathrm{mg} / \mathrm{dl})$.

Fue realizada ecografía abdominal de urgencia que mostró gran masa heterogénea de $16 \mathrm{~cm}$ de diámetro dependiente de glándula suprarrenal derecha con compresión de vena cava, sin poder discernir si se trataba de hematoma o absceso. La TAC confirmó tal hallazgo, informándolo como hematoma con signos de sangrado activo, de $16 \mathrm{~cm}$ de diámetro que comprimía y desplazaba vena cava (Fig. 2). Se indicó laparotomía urgente en la que se realizó suprarrenalectomía derecha y exéresis del hematoma (Fig. 3). El postoperatorio transcurrió sin incidencias y fue dado de alta al décimo día.

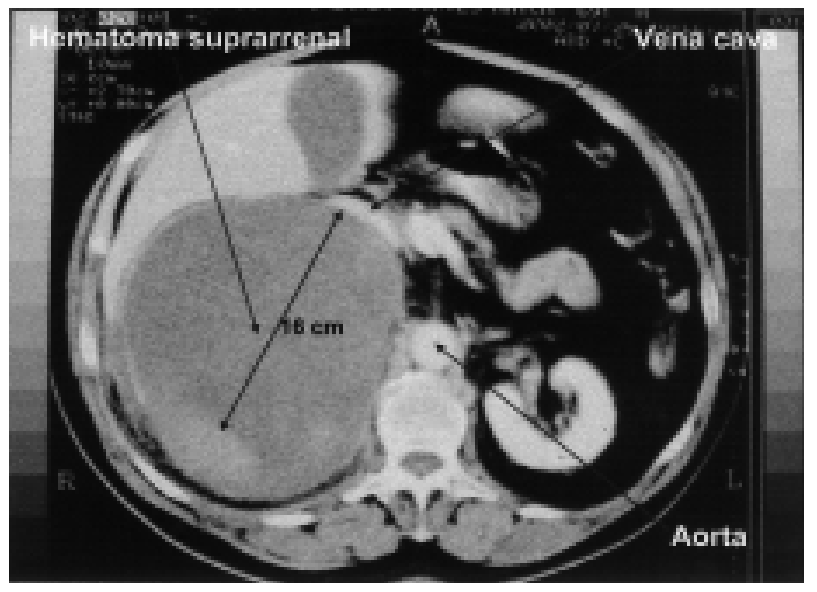

FIGURA 2. TAC de urgencia que muestra hematoma retroperitoneal dependiente de glándula suprarrenal derecha y vena cava rechazada anteriormente. 


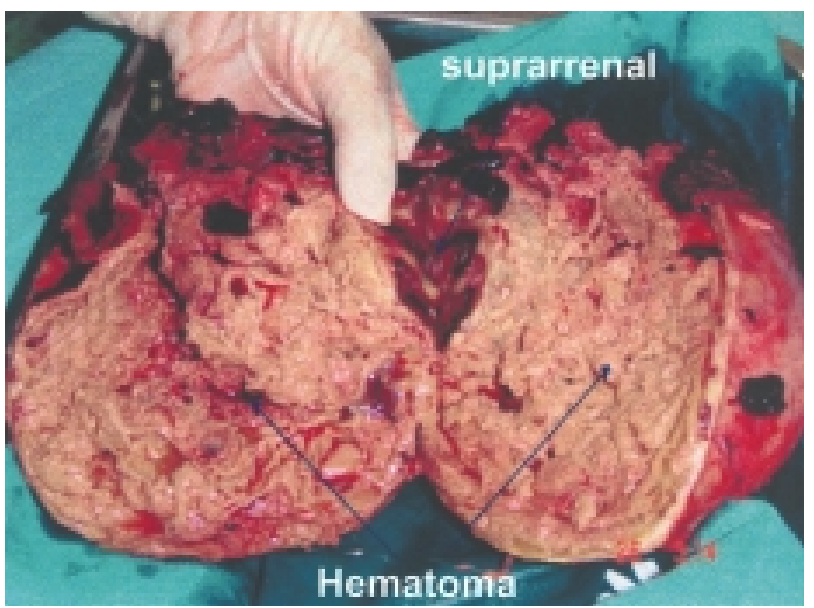

FIGURA 3. Hematoma y glándula suprarrenal tras su exéresis quirúrgica. El aspecto cavernoso y organizado del hematoma hacen suponer largo tiempo de evolución.

\section{DISCUSIÓN}

Etiológicamente, la hemorragia retroperitoneal puede ser clasificada en espontánea, o secundaria a traumatismos externos y maniobras endourológicas o endovasculares.

La primera, se produce como consecuencia de patología local o sistémica. Entre las sistémicas, encontraríamos las alteraciones de la coagulación (terapia con anticoagulantes orales o heparina, leucemia, policitemia, hemofilia, sindrome antifosfolípidos) y vasculitis, destacando la panarteritis nodosa, por rotura de aneurismas originados por el depósito de inmunocomplejos en las paredes de arterias de pequeño y mediano calibre $^{4-6}$.

Dentro de las causas locales, podemos distinguir las de origen renal, suprarrenal u otros órganos retroperitoneales, destacando entre todas ellas, por ser la más frecuente, la rotura de aneurisma de aorta abdominal. Con respecto a las renales (síndrome de Wünderlich), el estudio de Oesterling, demostraría que el angiomiolipoma, por su amplia vascularización, es la lesión con mayor tendencia al sangrado (51\%), pero sin superar, en valores absolutos, el adenocarcinoma ${ }^{5}$. Mencionar también, como causas menores habituales, pielonefritis, abscesos, nefritis, enfermedades vasculares renales e incluso rechazo agudo o crónico de riñones trasplantados ${ }^{4}$.

$\mathrm{El}$ origen suprarrenal es poco frecuente y se asocia a situaciones de stress (sepsis, cirugía, grandes quemados, gestaciones complicadas,...) $\mathrm{y}$, muy infrecuentemente, tratamiento prolongados con corticoides y ACTH. En todos estos casos, la hemorragia suele ser bilateral ${ }^{5}$.

Determinados tumores suprarrenales, también pueden ocasionar hemorragia retroperitoneal. Obviamente, en estos casos, el hematoma será unilateral. Los más frecuentemente asociados son feocromocitoma, adenoma, carcinoma, lesiones metastásicas y mielolipoma ${ }^{4,5}$.

En nuestro caso, consideramos que el mielolipoma fue la causa y el traumatismo factor desencadenante, pues este último, por si mismo, no justifica el hematoma hallado.

En relación a la clínica del $\mathrm{HR}$, si bien los autolimitados y de pequeño tamaño suelen ser asintomáticos, los sintomas y signos más frecuentemente descritos son dolor lumboabdominal, masa abdominal palpable y anemia. Los dos primeros y el shock hipovolémico constituyen la triada de Lenk, que se da, aproximadamente, en el $30 \%$ de los pacientes ${ }^{5}$. En nuestro caso se encontraban presentes el dolor abdominal y la anemia no siendo posible palpar el hematoma, probablemente, por tratarse de un abdomen globuloso y distendido. La no existencia de shock hipovolémico, pudiera deberse a la contención del hematoma por la fascia de Gerota.

El diagnóstico de confirmación del HR debe basarse en las pruebas de imagen. La ecografía es, sin duda, útil para el diagnóstico inicial por su rapidez de realización, inocuidad y elevada sensibilidad. Sin embargo, resulta menos eficaz, y por tanto específica, para definir la naturaleza del proceso ${ }^{4,5,7}$. De hecho, en nuestro caso, no fue posible excluir un origen neoplásico o incluso absceso.

La arteriografía estaría indicada si sospechamos una PAN para objetivar los aneurismas ${ }^{4}$. La TAC, hoy día, constituye la prueba de imagen princeps en el diagnóstico, tanto del HR como del mielolipoma, desplazando a otras como la pielografía intravenosa ${ }^{7}$. Aproximadamente, el $2 \%$ de los TAC realizados en politraumatizados demuestra hematoma de glándula suprarrenal ${ }^{8}$. En nuestro caso, resultó ser la prueba más ventajosa, pues confirmó que se trataba de hematoma, delimitó con mayor precisión la compresión de estructuras vecinas, y evidenció la existencia 
de sangrado activo. La RMN no parece aportar ventajas sobre la TAC, de forma que sólo suele realizarse cuando esta última no resulta concluyente ${ }^{7}$.

La compresión de estructuras vecinas por hematoma suprarrenal es muy poco frecuente, y se produce cuando son de gran tamaño, constituyendo uno de los criterios de indicación quirúrgica. En hematomas de glándula suprarrenal derecha, por su estrecha relación anatómica, las estructuras más afectadas suelen ser el riñón ipsilateral, hígado y vena cava infrahepática ${ }^{8}$.

En nuestro caso, y como muestran las imágenes aportadas, la vena cava se encontraba desplazada anteriormente hasta el punto de colapsarla. A pesar de ello, el paciente no presentaba edemas en miembros inferiores ni otra sintomatología secundaria a dicha compresión.

El tratamiento del HR dependerá del estado hemodinámico del paciente. Ante inestabilidad, las primeras medidas deberán ir orientadas a la reposición de la volemia y corrección de las posibles alteraciones de la coagulación ${ }^{9}$. Nuestro paciente, no se encontraba en situación de shock, posiblemente, y como mencionamos anteriormente, por la contención ejercida por la fascia de Gerota. No obstante, el gran tamaño del mismo y el sangrado activo, objetivado en la TAC, hacian peligrar la integridad de la misma, existiendo, por tanto, importante riesgo de hemorragia retroperitoneal masiva. Esto, y el desplazamiento y colapso de vena cava, nos orientaron a indicar laparotomía urgente. Se valoró la posibilidad de realizar embolización angiográfica para controlar el sangrado activo, pero fue desechada dado que no habría resuelto la compresión de vena cava.
En general, el tratamiento cabe decir que, en ausencia de complicaciones, debe ser conservador, manteniendo actitud expectante. En aquellos hematomas con signos de sangrado activo puede estar indicada la embolización angiográfica ${ }^{9}$. La cirugía debe limitarse a pacientes hemodinamicamente inestables (una vez descartada o fracaso de embolización), existencia o riesgo de hemorragia masiva y compresión de estructuras nobles ${ }^{4,5,9}$.

\section{REFERENCIAS}

1. Rodríguez JM, Parrilla P, Piñero A. Incidentaloma Suprarrenal. In: Sitges. Serra A, Sancho Insenser JJ. Cirugía Endocrina. Madrid: Aran S.A. 1999; 161-164.

2. Page D, DeLellis R, Hough A. Tumors of the Adrenal. In: Washington D.C. William H, Hartaman D. Atlas of tumor Pathology. Armed Forces Institute. 1985;162-166.

3. Amano T, Takemae K, Niikura S, et al. Retroperitoneal hemorrhage due to spontaneous rupture of adrenal myelolipoma. Int J Urol 1999;6(11):585-588.

4. Machuca J, Julve E, Galacho A, et al. Spontaneous retroperitoneal hematoma: our experience. Actas Urol Esp 1999;23(1): 43-50.

5. Vendrell JR, Alcover J, Alcaraz A, et al. Hematoma suprarrenal espontáneo unilateral, una causa excepcional de hemorragia retroperitoneal. Actas Urol Esp 1996;20(1):59-62.

6. Granry JC, Houet JF, Delhumeau A. Hematoma of the adrenal glands and heparin. Ann Fr Anesth Reanim. 1989;8(6):650655.

7. Kawashima A, Sandler CM, Ernst RD, et al. Imaging of nontraumatic hemorrhage of the adrenal gland. Radiographics. 1999;19(4):949-963.

8. McAlinden MG, McCrory DC, Diamond T. Post-traumatic adrenal haematoma with massive adrenal enlargement and compression of the vena cava. Br J Urol 1994;73(4):463-464.

9. Hendrickson RJ, Katzman PJ, Queiroz R, et al. Management of massive retroperitoneal hemorrhage from an adrenal tumor. Endocr J 2001;48(6):691-696.

Dr. G. Suárez Artacho

C/ Virgen de la Cinta 21; $3^{\circ} \mathrm{C}$

41011 Sevilla

(Trabajo recibido el 15 diciembre de 2003) 